\title{
Effectiveness of a 6-Month Isoniazid on Prevention of Incident Tuberculosis Among People Living with HIV in Eritrea: A Retrospective Cohort Study
}

\author{
Mulugeta Russom (D) - Henok G. Woldu (D) - Araia Berhane (D) · \\ Daniel Y. B. Jeannetot · Bruno H. Stricker (D) · Katia Verhamme (iD
}

Received: November 9, 2021 / Accepted: January 7, 2022 / Published online: January 29, 2022

(C) The Author(s) 2022

\section{ABSTRACT}

Introduction: A 6-month isoniazid as tuberculosis preventive therapy (TPT) for people living with HIV (PLHIV) was nationally introduced in Eritrea in 2014. However, its effectiveness in

Supplementary Information The online version contains supplementary material available at https:// doi.org/10.1007/s40121-022-00589-w.

M. Russom ( $\square)$

Eritrean Pharmacovigilance Centre, National

Medicines and Food Administration, Ministry of

Health, Asmara, Eritrea

e-mail: satiswt@gmail.com;

m.russomghebremedhin@erasmusmc.nl

M. Russom - D. Y. B. Jeannetot - K. Verhamme

Department of Medical Informatics, Erasmus

Medical Centre, Rotterdam, The Netherlands

H. G. Woldu

The Center for Health Analytics for National and Global Equity, Columbia, MO, USA

\section{A. Berhane}

Communicable Disease Control Division,

Department of Public Health, Ministry of Health, Asmara, Eritrea

B. H. Stricker

Department of Epidemiology, Erasmus Medical Centre, Rotterdam, The Netherlands

\section{Russom}

European Program for Pharmacovigilance and Pharmacoepidemiology, University of Bordeaux, Bordeaux, France preventing tuberculosis (TB) and duration of protection was questioned by physicians. This study was, therefore, conducted to evaluate the impact of the isoniazid preventive therapy (IPT) primarily on the prevention of TB and duration of its protection in PLHIV.

Methods: A retrospective cohort study was conducted that selected all eligible PLHIV attending HIV care clinics in all national and regional referral hospitals in Eritrea. Data was collected from patients' clinical cards using a structured data extraction sheet. The association between use of IPT and outcomes of interest was assessed using a Cox proportional hazard regression model and Kaplan-Meier curve.

Results: A total of 6803 patients were selected, which accounted for $75 \%$ of all PLHIV-accessing HIV care clinics in Eritrea. About $76 \%$ of patients were exposed to IPT while the remaining $24 \%$ were unexposed. The mean follow-up time was 4.9 years (SD 1.4). The incidence rate of TB was 1.7 and 10 cases per 1000 person-years in the exposed and unexposed, respectively. The unexposed had a higher risk of incident TB (adjusted hazard ratio [aHR] 3.75, 95\% confidence interval $[\mathrm{CI}] 2.89,6.13)$ and all-cause mortality (HR 2.41, 95\% CI 1.85, 3.14) compared to the exposed. A Kaplan-Meier curve showed that the exposed group had a higher TBfree follow-up probability (98.8\%) compared to the unexposed (95\%) at 65 months of follow-up $(p<0.001)$. IPT protection decreased rapidly 6 months after isoniazid completion. 
Conclusion: Use of a 6-month isoniazid as TPT was found to be effective in reducing incident TB in PLHIV-accessing HIV care clinics in Eritrea. However, the protection appeared to diminish soon, namely 6 months after completion of isoniazid, which warrants immediate attention from policy makers.

Keywords: All-cause mortality; Duration of protection; Eritrea; Isoniazid; People living with HIV; Prevention of TB; Tuberculosis preventive therapy

\section{Key Summary Points}

Why carry out this study?

In Eritrea, there was an assumption that a 6-month course of isoniazid as tuberculosis (TB) preventive therapy could protect development of active TB in people living with HIV (PLHIV) for about 5 years.

Effectiveness of isoniazid in preventing TB for PLHIV and the duration of protection have been questioned by physicians working in HIV care clinics that affected the uptake/implementation of the preventive therapy.

This study was conducted to evaluate the impact of isoniazid preventive therapy (IPT) on the prevention of TB and its duration of protection in PLHIV.

\section{What was learned from the study?}

This large real-world observational cohort study carried out in a moderate-TB burden country confirmed that the 6-month course of IPT was found to be effective in reducing incident $\mathrm{TB}$.

The duration of prevention, nevertheless, appeared to diminish rapidly after 6 months following completion of the intervention which warrants attention from policy makers and/or program directors on how to ensure patients' longterm protection from developing active TB.

\section{INTRODUCTION}

Tuberculosis (TB) is one of the main opportunistic infections in people living with HIV (PLHIV) and reports show that the risk of developing TB in PLHIV is 20-37 times higher than in those without HIV [1]. It was reported that PLHIV also have a significantly increased risk of developing extrapulmonary TB (40-80\%) compared to those without HIV (10-20\%) [2]. Furthermore, TB-related mortality accounts for $26 \%$ of the globally reported HIV deaths [3]. It was estimated that about one-fourth of the world's population is latently infected with mycobacterium tuberculosis [4]. In PLHIV, the lifetime risk of progression from latent $\mathrm{TB}$ infection to active disease is about $30 \%$ compared to the $10 \%$ risk in the general population [5].

Among the various options, the World Health Organization (WHO) recommends use of a 6-36-month course with isoniazid (INH) as tuberculosis preventive treatment (TPT) to reduce the incidence of active TB in PLHIV [6]. Several studies of different designs, including a meta-analysis, conducted between 2014 and 2020, confirmed the considerable protective effect of isoniazid preventive therapy (IPT) in PLHIV compared to those unexposed [7-14]. A systematic review and meta-analysis of randomized placebo-controlled trials reported that IPT has considerable benefits regardless of the results of tuberculin skin test (TST) [12]. On the contrary, two randomized controlled trials (RCTs) and a previously conducted systematic review, in children, reported that IPT has no beneficial effect on prevention of TB in PLHIV [15-17]. Conclusions on the impact of IPT on all-cause mortality have, however, been conflicting [7, 12, 13, 18, 19].

In Eritrea, INH prophylaxis for children under 5 years of age who are household contacts of bacteriologically confirmed pulmonary TB has been one of the pillars of TB prevention for at least two decades. A 6-month INH as TPT for PLHIV was, however, programmatically introduced in 2014. Following its introduction, with massive rollout, the intervention was taken by several PLHIV until the uptake and 
coverage were affected by INH-related adverse reactions and other unknown factors. As a result, the effectiveness of INH in preventing TB and duration of its protection have been an issue for physicians and PLHIV. To evaluate the effectiveness-risk of IPT, three parallel studies were carried out to measure the effectiveness of a 6-month isoniazid, risks of IPT, and factors that limit healthcare providers' acceptability of IPT. This study was, therefore, conducted primarily to evaluate the impact of IPT on the reduction of $\mathrm{TB}$ incidence and duration of its protection in PLHIV in Eritrea. A secondary objective was to measure the effect of IPT in reduction of all-cause mortality. Eritrea, with an estimated population of 3,546,000 [20], has a TB incidence of 67 cases per 100,000 inhabitants per year and 8957 PLHIV receiving antiretroviral therapy [21]. At the end of 2019, the adult HIV prevalence was estimated to be $0.7 \%$ in women and $0.4 \%$ in men [22].

\section{METHODS}

\section{Study Design and Setting}

This was a retrospective cohort study that involved all HIV care clinics of the national and regional referral hospitals (including private ones) in Eritrea. These clinics provide services to over $75 \%$ of PLHIV-accessing HIV care services in the country [20]. The hospitals involved in the study were Orotta national referral and teaching hospital, Orotta pediatric teaching hospital, Halibet national referral hospital, Sembel and Hazhaz Hospitals from the Central (Maekel) zone as well as Mendefera, Keren, Assab, and Barentu zonal referral hospitals and Massawa hospital, one hospital from each of the remaining five administrative zones of the country. Data for the study was collected between August 31 and November 1, 2020, by exploring all patients' medical cards of all people who received IPT and antiretroviral therapy (ART) as well as ART only at any time during the study period, including medical cards of patients who died during the study period.

\section{Study Population}

All PLHIV who attended the aforementioned HIV care clinics between November 1, 2014 (official start date of IPT for PLHIV in the country) and November 1, 2020 were considered as candidates for the study regardless of their IPT exposure status. As referral centers, these HIV care clinics offer services to PLHIV residing in different geographic regions of the country. All patients, regardless of their age and sex, who had at least 1 year of recorded followup (for both exposed and unexposed groups) and had no active $\mathrm{TB}$ at the time of first enrollment into the study were included in the study to enable us compare survival without incident TB. Hence, as the minimum follow-up time of exposure for both groups (ART \& IPT vs ART only) was 1 year, all the exposed group entered the study after completion of the 6-month IPT. Individuals diagnosed with incident TB anytime during the 6-month IPT were included into the study if they had a recorded follow-up of at least 1 year. Patients who died while on the 6-month IPT were excluded as the study aimed to measure the effect of a 6-month isoniazid on the prevention of incident TB. Individuals exposed to IPT and/or ART for less than 1 year during the study period and those who interrupted the 6-month IPT for any reason and could not complete the regimen were excluded for the aforementioned reason. PLHIV transferred in from other treatment sites and who completed a 6-month IPT but had no data on IPT start date were also excluded from the study as there was no information on whether the outcome developed before or after initiation of IPT. Moreover, those unexposed to ART during follow-up period (unexposed groups with no ART), lost to follow-up, died, or transferred out before completion of the 6-month course of IPT and/or diagnosed with active TB before starting ART or IPT (during the study period) were excluded as the study aimed to measure the effectiveness of a 6-month IPT. Recruitment of patients who started but failed to complete a 6-month course of IPT as if they were exposed or unexposed could introduce bias; thus, such defaulters of IPT were excluded from both groups. 


\section{Data Sources}

Available non-electronic (paper-based) patient registers and clinical cards were used as the data sources for this study. Information on patients' sociodemographic characteristics, clinical and laboratory data, history of frequent alcohol use, smoking, imprisonment, malnutrition, and comorbidities were obtained from these sources. The data sources also provided additional details such as type of ART regimen, cumulative exposure time to ART, duration and history of interruption of IPT, previous history of TB, treatment adherence to ART and IPT, baseline CD4 cell count, HIV viral load, incident TB, method of diagnosis, time between initiation of IPT and incident TB, follow-up status, and date and cause of death. Adherence to ART and/or IPT was captured from patients' medical cards. In the medical cards, patients' level of adherence was marked as "good", "fair", and "poor" based on certain parameters [see Table S1 in the Electronic Supplementary Material].

\section{Exposure and Outcome Measurement}

The main exposure of interest was confirmed completion of a 6-month course of daily INH $300 \mathrm{mg}$ tablet for adults and $10 \mathrm{mg} / \mathrm{kg}$ for children as TB preventive therapy. On the basis of the clinical records, PLHIV who had ever completed the 6-month course of IPT during the study period were considered as exposed and those who never took IPT were categorized as non-exposed. Both the exposed and unexposed groups were on ART, while the exposed group were also receiving adjuvant vitamin $B_{6}$ tablets.

The primary endpoints were incident $\mathrm{TB}$, diagnosed according to the national guideline at any time during the study period, duration of protection of TB by IPT, and TB-free follow-up probability [23]. Documented incident TB diagnosed with GeneXpert and/or acid-fast bacilli or clinically or culture were considered as TB cases. As per the Comprehensive HIV/AIDS Care Manual, Eritrea [23], PLHIV are routinely (at every visit) screened for TB using a clinical algorithm. Patients with any symptoms of current cough, night sweats, weight loss, and fever are evaluated using sputum microscopy, chest $\mathrm{X}$-ray, GeneXpert, or culture as appropriate. Though the protocol was not primarily designed to measure the effect of IPT on allcause mortality, the authors considered it as a secondary outcome measure with some limitations. Mortality information and cause of death were taken as documented in patients' medical cards. As a routine practice, once a patient died, the consulting physician tries to assess the cause of death and document their impression in the medical cards. For patients reported to be deceased at home or outside a health facility, the cause of death was recorded in their medical cards as "unknown" if not otherwise clear enough, such as "car accident". As reported in the exclusion criteria, all patients enrolled into this study definitely survived for 1 year and thus, to avoid immortal time bias, 12 months was subtracted from the cumulative follow-up time of each study participant, including the IPT exposure time for those who had less than 18 months of overall follow-up time and computed accordingly. As for the analysis of the effect of IPT on reduction of TB-related mortality, only those who had TB as the only documented cause of death were considered.

\section{Data Collection Tools and Patient Enrollment Approach}

A structured data extraction sheet prepared for this purpose was used to collect historical data of patients from the HIV care clinics [see Table S2 in the Electronic Supplementary Material]. The data collection tool comprises the study participants' sociodemographic characteristics, treatment or exposure details, and treatment outcomes. At each study site, two data collectors were recruited and a 1-day orientation workshop was provided to familiarize the data collectors with the study objectives, data collection approach, and data collection tools. Prior to data collection, the data extraction sheet was circulated, for content validity, to five different experts from the fields of pharmacy, clinical pharmacy, medicine, and epidemiology. Accordingly, the questions that were marked as "irrelevant" by the majority of 
the reviewers were either adjusted or removed. A 3-day pre-test was carried out in one hospital collecting historical data and the information gathered was used to improve comprehensiveness of the data collection tool, ensure consistency of the data abstraction process, and data collection approach.

The data collectors screened all clinical cards of PLHIV for eligibility before starting the enrollment process and cases not fulfilling the eligibility criteria were excluded right from the onset of the study (Fig. 1). Excluded cases were documented in a listing form to identify reasons for exclusion [see Table S3 in the Electronic Supplementary Material]. For the eligible cases, patients' clinical cards, registers, and laboratory results were reviewed and the required information was documented in the structured questionnaire as appropriate. Eligible PLHIV that started their ART before November 1, 2014, start to contribute person-time since November 1, 2014 and stop to contribute on the day they develop incident TB, where applicable. The reason for considering November 1, 2014 to be the study start date is because it was during that time that IPT was officially deployed in Eritrea.

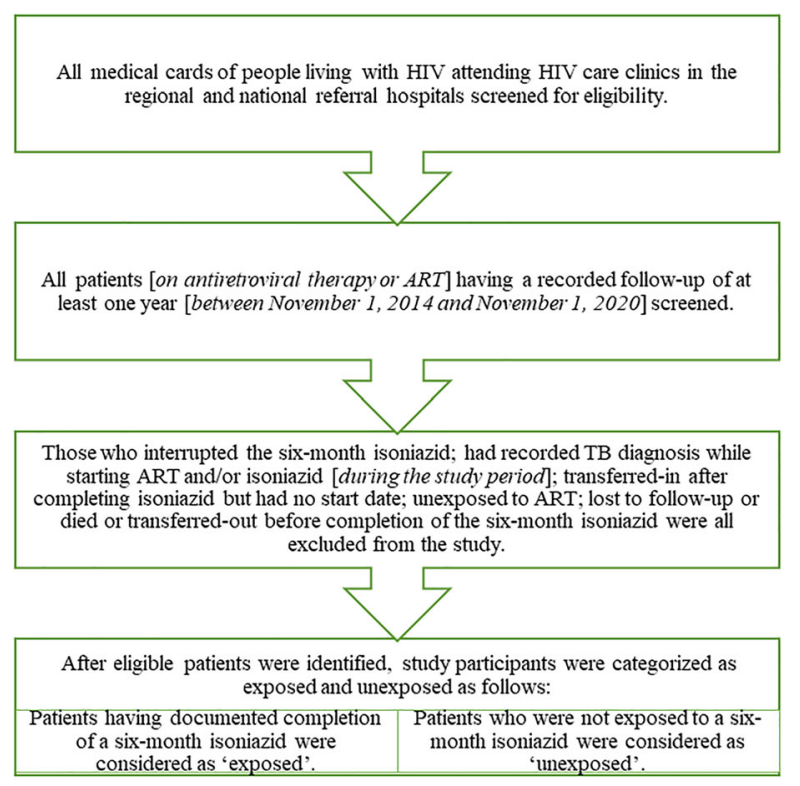

Fig. 1 Study participants selection process on effectiveness of a 6-month isoniazid preventive therapy (IPT) in people living with HIV in Eritrea conducted between November 1, 2014 and November 1, 2020
For unexposed patients diagnosed with HIV and/or started IPT any time after November 1, 2014, they contributed person-time from the date they start ART and/or IPT until November 1, 2020 or they developed the outcome of interest. Study participants who were lost to follow-up, died, or developed incident TB any time during the follow-up period stopped to contribute person-time by the time they encounter these events. The exposed group were starting IPT a few months after they start ART. Thus, none of the members of the exposed group had contributed person-time to the unexposed group. The time at which the first diagnosis of TB was documented, during the study period, was considered as the index date.

\section{Statistical Analysis}

Data was entered in CSPro 7.4 and analyzed using R 4.0.4 [24], and SPSS 20.0. Data cleaning was carried out manually and in SPSS using several counterchecking items. Descriptive statistics including percentages, frequencies, number needed to treat, mean (SD), and median (IQR) were computed as appropriate. Participants with missing data in specific variables of interest such as history of imprisonment, close contact with a patient with $\mathrm{TB}$, malnutrition, smoking, regular alcohol use, and so on were not included in the analysis of a specific variable. The incidence rate of TB was computed using descriptive statistics and, as the cohort was dynamic, the incidence rate of active TB in exposed and unexposed with person-time (years) in the denominator was used as a measure of frequency. As step 1, both chi-square test of independence and bivariate logistic regression were used to identify factors that are associated with incident TB. Variables that were found to have significant associations in the univariate analysis were then fitted into a Cox proportional hazard model to control for confounders and analyze the protective effect of IPT. A survival regression analysis model was used to compute the time to onset of incident $\mathrm{TB}$ (the time between the start of the follow-up time and development of incident TB) and survival probability. Kaplan-Meier estimator 
was used to create graphs of the observed survival curves or survival times without TB and log-rank test was used to compare survival probability between the exposed and unexposed groups. Rate ratio or hazard ratio with 95\% confidence interval was used as a measure of association or impact of IPT on the prevention of $\mathrm{TB}$, survival without $\mathrm{TB}$, and all-cause mortality. To further explore the effect of IPT on reduction of $\mathrm{TB}$ in children, subgroup analysis was carried out. For all statistical tests, a twosided alpha level of 0.05 was used to determine statistical significance.

\section{Ethical Considerations}

Ethical clearance to conduct the study was obtained from the Health Research Ethics and Protocol Review Committee of the Ministry of Health of the State of Eritrea (reference number 7-18/2020). Patients' informed/written consent was waived because of the retrospective nature of the study. The study followed the latest version of the Helsinki Declaration.

\section{RESULTS}

\section{Sociodemographic Characteristics}

A total of 8028 PLHIV enrolled from HIV care clinics were screened for eligibility, out of whom 6803 met the study inclusion criteria (Fig. 2). The majority of the study population were female $(63.6 \%)$ and urban residents (75.6\%). The median age, taken at enrollment, was 40 years (interquartile range [IQR] 14; range 1-85 years). About $12 \%$ had no formal education and $65.3 \%$ were unemployed. Known history of smoking and frequent alcohol use was reported in only $5.1 \%$ and $3.2 \%$, respectively. About 4\% (281/6308) of all participants had at least one chronic comorbidity and $5.4 \%$ had a previous history of TB. During the study period, $3.7 \%$ of the study participants had a history of at least one imprisonment and $1.9 \%$ had a history of close contact with a person having TB. Detailed sociodemographic characteristics of the study population are listed in Table 1.
The majority of the study participants (71.3\%) were attending health facilities located in Maekel zone, Asmara, the capital of Eritrea. About $14.3 \%$ of PLHIV attending HIV care clinics located in Maekel zone (Central region) and $50 \%$ of those in other zones were unexposed to IPT (Table 2).

At initiation of IPT, the unexposed group were more frequently younger (median age 37 years [IQR19]; $p<0.0001)$, had body weights less than $51 \mathrm{~kg}(p<0.0001)$, were unemployed $(p<0.0001)$, had higher proportions of patients with no formal education $(p<0.0001)$ and higher proportions of subjects having a history of malnutrition $(p=0.029)$ and a baseline CD4 cell count of less than 200 cells $/ \mu \mathrm{L}(p<0.0001)$. On the other hand, the exposed group had a higher proportion of patients with urban residence $(p<0.0001)$, history of imprisonment $(p=0.045)$, previous history of TB $(p<0.0001)$, undetectable viral load $(p<0.0001)$, good level of adherence to ART $(p<0.0001)$, and longer mean follow-up time $(p<0.0001)$ (Tables 1 and $3)$.

\section{Clinical and Laboratory Follow-Up}

The mean and median cumulative exposure time to ART (exposed and unexposed patients altogether) was 8.6 years (SD 3.8) and 8.8 years (IQR 6.3), respectively (Table 1 ). In general, $75.6 \%$ of the study participants were exposed to IPT while the rest were unexposed (Fig. 2). The majority of the study participants $(72.6 \%)$ were on efavirenz-based ART regimen and were observed from 1 to 6 years with a mean followup time of 4.9 years (SD 1.4). Their level of adherence to ART was marked as "good" in $91.2 \%$ (Table 3 ). About $80 \%$ of study participants were still on follow-up by the time the study was completed and all-cause mortality proportion was $3.6 \%$. About three-quarters (74.4\%) of the study participants had undetectable viral load and $86.4 \%$ had CD4 cell count greater than 200 cells $/ \mu \mathrm{L}$ while $39.4 \%$ had greater than 500 cells $/ \mu \mathrm{L}$ (Table 1 ). By the time of completion of the study, about $98.1 \%$ of the exposed and $87.7 \%$ of the unexposed were on ART for at least 3 years. Overall, the follow-up 


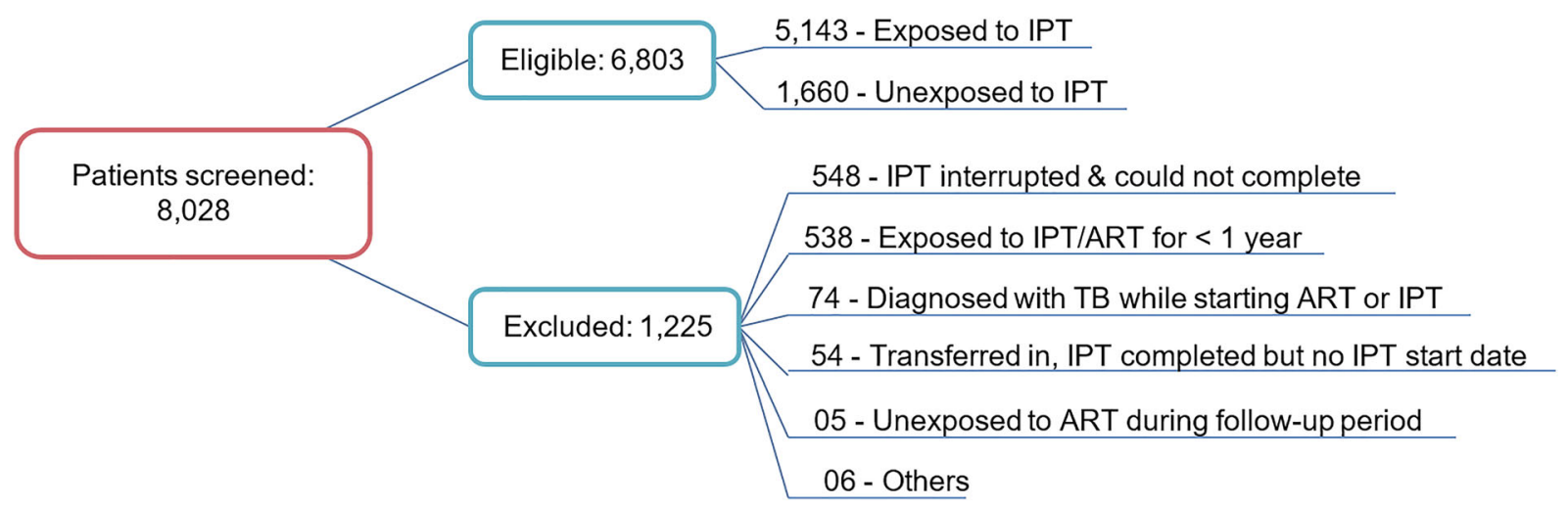

Fig. 2 Summary of enrolled patients and eligibility criteria for the assessment of isoniazid preventive therapy (IPT) effectiveness for subjects followed up between November 1, 2014 and November 1, 2020

period for exposed and unexposed groups was 26,398 and 6737 person-years, respectively.

\section{Incidence Rate of Tuberculosis and its Associated Factors}

During the 6-year follow-up period, 0.9\% (44/ $5,143)$ of the exposed and $4.1 \%(68 / 1660)$ of the unexposed patients developed incident TB with an overall cumulative incidence of $1.7 \%$. As patients had different lengths of risk window, incidence rates of TB were calculated. The incidence rate in the exposed was about 1.7 cases per 1000 person-years whereas in the unexposed it was 10 cases per 1000 person-years (Table 4). The method of diagnosis for TB was $58 \%$ bacteriologically confirmed (acid-fast bacilli positive, 41; GeneXpert, 22; and culture, 2 ) while the rest were clinically diagnosed.

During the first 6 months of follow-up, no incident TB was reported in the exposed group whilst in the unexposed group there were $31 \mathrm{~TB}$ cases within this time frame. Eight of the 44 incident $\mathrm{TB}$ cases $(18.8 \%)$ observed in the exposed cohort were documented right after the completion of the 6-month INH preventive therapy (first 7-12 months) (Fig. 3, Table 4). The median time to onset of incident $\mathrm{TB}$ was 28.5 months (IQR 30) in the exposed group and 10.5 months (IQR 30) for the unexposed.
Univariate analysis showed that those unexposed to IPT were more likely to develop TB compared to those exposed. Moreover, being female, history of regular alcohol use, history of malnutrition (undernourished), history of imprisonment, baseline CD4 count less than 200 cells $/ \mu \mathrm{L}$, detectable HIV viral load, poor or fair adherence to ART, shorter cumulative exposure to ART, and shorter cumulative follow-up time were all associated with a higher incidence rate of TB (Table 5).

After candidate variables were fitted to a multivariate Cox proportional hazard model, those unexposed to IPT were at higher risk of developing TB compared to the exposed (aHR $3.75,95 \%$ CI $2.89,6.13)$. Other variables such as having lower body weight (aHR 2.00, 95\% CI $1.18,3.39$ ), history of malnutrition (aHR 5.74, 95\% CI 2.14, 15.33), history of imprisonment (aHR 2.97, 95\% CI 1.28, 6.92), lower CD4 cell count (aHR 1.92, 95\% CI 1.11, 3.32), and decreased adherence to ART (aHR 2.44, 95\% CI $1.18,5.02)$ were still found to be associated with an increased risk of TB (Table 6). Sex, history of regular alcohol use, CD4 cell count, cumulative exposure to ART, and cumulative follow-up time all lost their statistical significance in the fitted multivariate model. 
Table 1 Sociodemographic characteristics of PLHIV, both exposed and unexposed cohorts to IPT, attending at all national and regional referral hospitals in Eritrea followed up between November 1, 2014 and November 1, $2020(N=6803)$

Variables (measured at baseline)

\begin{tabular}{ll} 
Cohort group & \\
\hline Exposed to IPT & Unexposed to IPT \\
$N=5143$ & $N=1660$ \\
$n$ & $\%$
\end{tabular}

$p$ value

Age (years) median (IQR) 40 (14), range 1-85

$\begin{array}{ll}<16 & 351(6.8) \\ 16-59 & 4547(88.4) \\ \geq 60 & 245(4.8)\end{array}$

$274(16.5)$

$<0.001$

Sex

Male

1896 (36.9)

$1311(79.0)$

Female

3247 (63.1)

$583(35.1)$

0.199

Educational status

No formal education

$522(10.1)$

$323(19.5)$

$<0.001$

Primary

$2491(48.4)$

861 (51.9)

Secondary

1888 (36.7)

407 (24.5)

Higher education

$242(4.7)$

$67(4.0)$

Employment status

Employed

2045 (39.9)

307 (18.8)

$<0.001$

Unemployed

3075 (60.1)

1329 (81.2)

Residence

Urban

3985 (78.4)

1103 (66.8)

$<0.001$

Rural

1097 (21.6)

$549(33.2)$

History of smoking

Yes

237 (5.4)

$59(4.1)$

0.053

No

4116 (94.6)

1365 (95.9)

History of regular alcohol use

Yes

No

$130(3.1)$

$4132(96.9)$

History of malnutrition

Yes

No

$29(0.6)$

$5060(99.4)$

History of imprisonment

Yes

$172(3.9)$

$4199(96.1)$
$52(3.9)$

0.146

$1297(96.1)$

$17(1.1)$

0.029

$1535(98.9)$

$39(2.8)$

0.045

$1363(97.2)$ 
Table 1 continued

Variables (measured at baseline)

\begin{tabular}{ll} 
Cohort group & \\
\hline Exposed to IPT & Unexposed to IPT \\
$N=5143$ & $N=1660$ \\
$n$ & $\%$
\end{tabular}

$p$ value

$N=1660$

History of close contact with a person having TB

$$
\text { Yes }
$$

No

Comorbidity

Yes

No

Type of comorbidities

Diabetes

$63(1.8)$

$3449(98.2)$

$225(4.4)$

$4918(95.6)$

$54(24.0)$

60 (26.7)

$17(7.6)$

$19(8.4)$

$20(8.9)$

$11(4.9)$

44 (19.6)

$311(6.0)$

$4832(94.0)$

606 (11.8)

4537 (88.2)

$\geq 200$

Viral load suppression (copies/mL)

Undetectable $(<50)$

Detectable $(\geq 50)$

Type of ART

Nevirapine based

Efavirenz based

Second line

Others
$2902(77.8)$

$828(22.2)$

1039 (20.2)

3856 (75.0)

$240(4.7)$

$8(0.2)$
$23(2.3)$

0.281

966 (97.7)

$56(3.4)$

0.075

$1604(96.6)$

$11(19.6)$

0.010

7 (12.5)

$2(3.6)$

7 (12.5)

2 (3.6)

7 (12.5)

$20(35.7)$

$54(3.3)$

$<0.001$

$1606(96.7)$

$321(19.3)$

$<0.001$

$1339(80.7)$

781 (64.2)

$<0.001$

$436(35.8)$

$487(29.4)$

$<0.001$

1080 (65.1)

$84(5.1)$

$8(0.5)$

$I P T$ isoniazid preventive therapy, $T B$ tuberculosis, $A R T$ antiretroviral therapy, $N$ number 
Table 2 Distribution of study participants by their exposure status and zones, Eritrea, 2020

\begin{tabular}{|c|c|c|c|c|}
\hline \multirow[t]{2}{*}{ Zones/regions } & \multicolumn{2}{|l|}{ Exposure status } & \multirow[t]{2}{*}{ Total } & \multirow{2}{*}{$\begin{array}{l}\text { Proportion of study participants } \\
\text { unexposed to IPT (\%) }\end{array}$} \\
\hline & Exposed (ART + IPT) & Unexposed (ART only) & & \\
\hline Maekel (Central) & 4159 & 692 & 4851 & 14.3 \\
\hline Debub & 272 & 216 & 488 & 44.3 \\
\hline Anseba & 372 & 275 & 647 & 42.5 \\
\hline Northern Red Sea & 155 & 50 & 205 & 24.4 \\
\hline Southern Red Sea & 132 & 5 & 137 & 3.6 \\
\hline Gash Barka & 53 & 422 & 475 & 88.8 \\
\hline Total & 5143 & 1660 & 6803 & 24.4 \\
\hline
\end{tabular}

$A R T$ antiretroviral therapy, IPT isoniazid preventive therapy

\section{Impact of IPT on Incidence of Tuberculosis}

After all other covariates were controlling for, IPT reduced the incidence of TB by $74 \%$ (95\% CI $60-83 \%)$ at about 73 months follow-up. Subgroup analysis aimed at exploring the effect of IPT on reduction of $\mathrm{TB}$ in children, aged 15 years and below, was carried out and the unexposed group had a higher risk of developing incident $\mathrm{TB}$ compared to those exposed (HR $4.39 ; 95 \%$ CI 1.20, 16.12). Besides, the number needed to treat (NNT) was found to be 30.8; meaning, at least about 31 PLHIV were needed to be treated with IPT in order to prevent incident TB in one patient.

\section{Survival Analysis}

Kaplan-Meier plot estimates showed that both the IPT exposed and unexposed groups had a good probability of TB-free follow-up, at least $95 \%$ at 65 months follow-up (Fig. 4). The mean TB-free follow-up time in the exposed and unexposed was 72.6 and 70.6 months, respectively (Fig. 4). In the exposed group, the median time to onset of incident TB was 28.5 months (IQR 30) and in the unexposed group the median was 10 months. About half of the cases (21/44) developed incident TB within 24 months following the start of IPT with as early as 7 months in a few patients (Fig. 3). At 65 months follow-up, those exposed to IPT had better probability of TB-free follow-up, over $98.8 \%$, compared to the unexposed, 95\%, $(p<0.001)$.

\section{Impact of IPT on All-Cause Mortality}

Overall 241 all-cause deaths, 12 of which were TB-related, were reported. About $34 \%$ of the allcause mortality were related to other opportunistic infections including pneumonia and $10.4 \%$ were unspecified cancer related. The cause of death was unknown in $34.7 \%$ of the cases and was recorded merely as "death at home". Those who were unexposed to IPT had an increased risk of all-cause mortality (unadjusted HR 2.41, 95\% CI 1.85, 3.14) and TB-related deaths (unadjusted HR 4.27, 95\% CI 1.32, 13.85) compared to the exposed (Table 7).

\section{DISCUSSION}

This study confirmed that the use of a 6-month IPT in PLHIV had a significantly beneficial effect on the reduction of TB morbidity. The intervention was also found to be effective in reduction of incident $\mathrm{TB}$ in children, aged 15 years and below. Taking into consideration the relatively low TB-HIV co-infection rate in Eritrea and the risk of IPT-induced hepatic 
Table 3 Follow-up outcome characteristics of PLHIV, both exposed and unexposed cohorts to IPT, attending at all national and regional referral hospitals in Eritrea followed up between November 1, 2014 and November 1,2020 $(N=6803)$

\begin{tabular}{|c|c|c|c|c|}
\hline \multirow[t]{2}{*}{ Variables } & \multicolumn{2}{|l|}{ Cohort group } & \multirow{2}{*}{$\begin{array}{l}\text { Total } \\
n(\%)\end{array}$} & \multirow[t]{2}{*}{$p$ value } \\
\hline & $\begin{array}{l}\text { Exposed to IPT } \\
n(\%)\end{array}$ & $\begin{array}{l}\text { Unexposed to IPT } \\
n(\%)\end{array}$ & & \\
\hline \multicolumn{5}{|c|}{ Level of adherence to ART } \\
\hline Good & $4782(93.1)$ & $1414(85.4)$ & $6196(91.2)$ & $<0.001$ \\
\hline Fair & $233(4.5)$ & $124(7.5)$ & $357(5.3)$ & \\
\hline Poor & $123(2.4)$ & $118(7.1)$ & $241(3.5)$ & \\
\hline \multicolumn{5}{|c|}{$\begin{array}{l}\text { Overall cumulative ex } \\
\text { the study period) }\end{array}$} \\
\hline $1-2$ & $96(1.9)$ & $205(12.3)$ & $301(4.4)$ & $<0.001$ \\
\hline $3-5$ & $691(13.4)$ & $453(27.3)$ & $1144(16.8)$ & \\
\hline$>5$ & $4356(84.7)$ & $1002(60.4)$ & $5358(78.8)$ & \\
\hline
\end{tabular}

Overall cumulative follow-up time of exposed and unexposed study participants in years; mean (SD) 4.9 (1.4), median (IQR) 5.7 (1.5), range $1-6$

$\begin{array}{llll}1-2 & 159(3.1) & 286(17.2) & 445(6.5)<0.001 \\ 3-4 & 654(12.7) & 479(28.9) & 1133(16.7) \\ 5-6 & 4330(84.2) & 895(53.9) & 5225(76.8)\end{array}$

Current follow-up status

$\begin{array}{llll}\text { Still on follow-up } & 4316(83.9) & 1121(67.5) & 5437(79.9) \\ \text { Transferred out } & 441(8.6) & 288(17.3) & 729(10.7) \\ \text { Lost to follow-up } & 248(4.8 & 146(8.8) & 394(5.8) \\ \text { Died } & 138(2.7) & 105(6.3) & 243(3.6) \\ \text { All-cause mortality } & & & 243(3.6) \\ \text { Yes } & 138(2.7) & 105(6.3) & 6560(96.4) \\ \text { No } & 5005(76.3) & 1555(93.7) & 6803(100.0) \\ \text { Total } & 5143(100.0) & 1660(100.0) & 0.001\end{array}$

PLHIV people living with HIV, IPT isoniazid preventive therapy, SD standard deviation, IQR interquartile range, $A R T$ antiretroviral therapy, $N / n$ number

injury $[25,26]$, the need for IPT has been seriously argued by physicians working in the HIV care clinics. Although overall effectiveness-risk analysis of IPT is required, the findings of this study show that PLHIV on ART can significantly benefit from the use of IPT even in settings with lower rates of TB-HIV co-infection. The beneficial effect of isoniazid in preventing/reducing incident TB in PLHIV found in this study was consistent with previously reported findings of a meta-analysis and systematic reviews $[12,13]$. Even though the effect of IPT on the reduction 
Table 4 Incidence rate of tuberculosis per 1000 personyears against time of follow-up in months following commencement of the study in those exposed to isoniazid preventive therapy (IPT) versus those unexposed

\begin{tabular}{l} 
Variable Unadjusted HR (95\% CI $) \quad p$ value \\
\hline Incident TB in the first 6 months follow-up $(N=6803)$ \\
Exposed $\quad \mathrm{N} / \mathrm{A}$ \\
Unexposed \\
Incident TB in the first 12 months follow-up $(N=6803)$ \\
Exposed $14.1(6.55,30.33)$ \\
Unexposed \\
Incident TB in $13-24$ months follow-up $(n=6759)$ \\
Exposed $2.34(1.00,5.47)$ \\
Unexposed \\
Incident TB in $>24$ months follow-up $(n=6737)$ \\
Exposed $\quad 3.31(1.82,5.94)$ \\
\hline
\end{tabular}

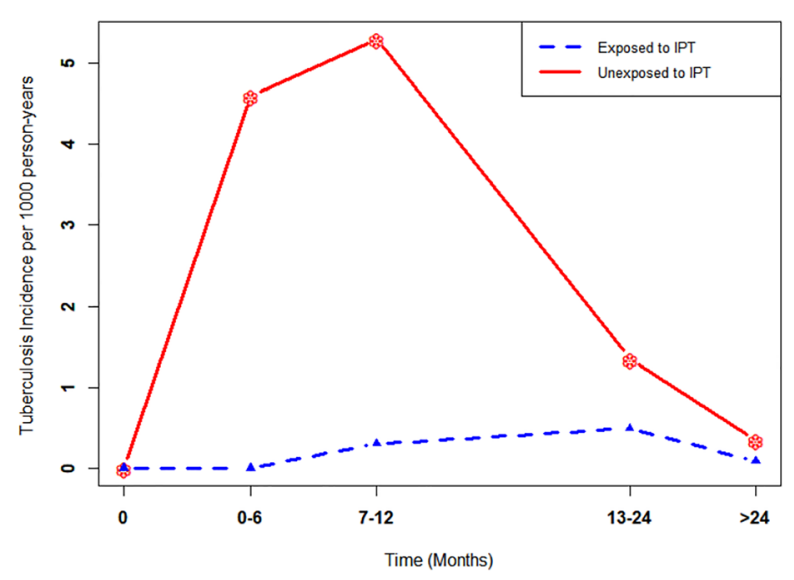

Fig. 3 Incidence rate of tuberculosis per 1000 person-years against time of follow-up in months following commencement of the study in those exposed to isoniazid preventive therapy (IPT) versus those unexposed

of all-cause mortality has been conflicting $[7,12,13,18,19]$, our current large observational study showed that those exposed to isoniazid had lower rates of all-cause mortality and TB-related mortality (Table 7). Readers should, however, cautiously interpret the results because of the small sample size in the subanalysis of the TB-related mortality and the cause of death was not known for about onethird of the overall mortalities. Moreover, the strength of evidence is limited as the protocol was not designed to measure the aforementioned outcomes. Another study with a focus on the impact of IPT on all-cause mortality could shed a more decisive light on that aspect.

There were a few studies that reported no benefits from IPT in PLHIV. To mention some, one study was conducted in children and another enrolled people with advanced HIV $[15,16]$. In the latter study, for instance, the fact that people with advanced HIV were likely to have severely weakened immunity, it might be difficult for IPT to significantly reduce the incidence of TB morbidity. Besides, that study had statistical power limitations due to small sample size.

Previously, there was an assumption that of PLHIV, only those having positive tuberculin skin test (TST) are likely to benefit from IPT [27-29]. Later on, other studies revealed that PLHIV regardless of their TST results can considerably benefit from IPT [30], and the WHO, in its revised IPT guideline, has clearly indicated that "TST is not a requirement for initiating IPT in PLHIV" [6]. In the current study, the TB preventive therapy was prescribed without TST and this finding supports the latter amendment that it can be beneficial even in the absence of TST which is a very important practicality aspect on IPT implementation.

Kaplan-Meier plot estimate showed that the exposed group had relatively better survival probability without TB or TB-free follow-up (ca. $98.8 \%)$ compared to the unexposed $(95.0 \%)$ at 65 months follow-up, which was statistically significant. It should, however, be noted that generally both the exposed and unexposed groups had good survival probability; thus, further studies are required to determine if such statistical significance is clinically meaningful/ significant taking the risks of the intervention into account. The reported duration of protection of IPT has been inconsistent, from several months to several years [31, 32], and in Eritrea, there was an assumption that a 6-month IPT 
Table 5 Incidence rate of tuberculosis and univariate analysis of associated factors in PLHIV attending at all national and regional referral hospitals in Eritrea followed up between November 1, 2014 and November 1, $2020(N=6803)$

\begin{tabular}{|c|c|c|c|c|}
\hline Variables & $\begin{array}{l}\text { Incident TB cases } \\
(n=112)\end{array}$ & $\begin{array}{l}\text { Incidence rate per } 1000 \text { person- } \\
\text { years }\end{array}$ & $\begin{array}{l}\text { Crude rate ratio } \\
(95 \% \mathrm{CI})\end{array}$ & $p$ value \\
\hline \multicolumn{5}{|c|}{ Exposure to IPT } \\
\hline Unexposed & 68 & $10.0(7.89,12.69)$ & $6.04(4.08,9.04)$ & $<0.001$ \\
\hline Exposed & 44 & $1.66(1.23,2.22)$ & Ref & \\
\hline \multicolumn{5}{|l|}{ Age (years) } \\
\hline$<16$ & 13 & $5.07(2.94,8.74)$ & $2.02(0.62,8.49)$ & 0.318 \\
\hline $16-59$ & 95 & $3.25(2.66,3.98)$ & $1.29(0.49,4.84)$ & 0.820 \\
\hline$\geq 60$ & 4 & $2.25(0.94,6.70)$ & Ref & \\
\hline \multicolumn{5}{|l|}{ Sex } \\
\hline Female & 59 & $4.89(3.62,5.42)$ & $1.96(1.33-2.90)$ & $<0.001$ \\
\hline Male & 53 & $2.24(5.87,8.92)$ & Ref & \\
\hline \multicolumn{5}{|l|}{ Residence $^{\$}$} \\
\hline Rural & 29 & $3.69(2.57,5.31)$ & $1.15(0.72-1.77)$ & 0.504 \\
\hline Urban & 81 & $3.22(2.59,4.00)$ & Ref & \\
\hline \multicolumn{5}{|c|}{ History of smoking ${ }^{\$, \Psi}$} \\
\hline Yes & 8 & $5.33(2.66,10.665)$ & $1.76(0.73,3.65)$ & 0.147 \\
\hline No & 74 & $3.03(2.41,3.80)$ & Ref & \\
\hline \multicolumn{5}{|c|}{ History of regular alcohol use ${ }^{\$, \Psi}$} \\
\hline Yes & 7 & $7.96(3.79,16.70)$ & $2.75(1.07,5.97)$ & 0.018 \\
\hline No & 70 & $3.10(2.50,3.84)$ & Ref & \\
\hline \multicolumn{5}{|c|}{ History of malnutrition $\$$} \\
\hline Yes & 6 & $29.00(13.02,64.53)$ & $9.30(3.33,20.96)$ & $<0.001$ \\
\hline No & 101 & $3.12(2.56,3.78)$ & Ref & \\
\hline \multicolumn{5}{|c|}{ History of imprisonment $t^{\$, \#}$} \\
\hline Yes & 8 & $8.01(4.00,16.0)$ & $2.48(1.04,5.13)$ & 0.021 \\
\hline No & 80 & $3.23(2.71,4.09)$ & Ref & \\
\hline \multicolumn{5}{|c|}{ Previous history of $\mathrm{TB}$} \\
\hline Yes & 7 & $3.67(1.74,7.69)$ & $1.09(0.43,2.34)$ & 0.687 \\
\hline No & 105 & $3.34(2.75,4.04)$ & Ref & \\
\hline \multicolumn{5}{|c|}{$\mathrm{CD} 4$ cell count $($ cells $/ \mu \mathrm{L})$} \\
\hline$<200$ & 39 & $9.76(7.13,13.36)$ & $3.92(2.59,5.87)$ & $<0.001$ \\
\hline$\geq 200$ & 73 & $2.49(1.97,3.13)$ & Ref & \\
\hline
\end{tabular}


Table 5 continued

Variables

$$
\begin{aligned}
& \text { Incident TB cases } \\
& (n=112)
\end{aligned}
$$

Incidence rate per 1000 personyears

$5.92(4.27,8.21)$

$3.04(2.34,3.93)$

$17.667(10.99,28.45)$

$9.99(66.12,166.31)$

$2.57(2.06,3.20)$

$1.74(0.25,12.38)$

$1.25(0.40,3.87)$

$1.66(1.20,2.27)$
Viral load suppression (copies/mL) ${ }^{\$,}$

Detectable $(\geq 50) \quad 36$

Level of adherence to ART

$\begin{array}{ll}\text { Poor } & 17 \\ \text { Fair } & 16 \\ \text { Good } & 79\end{array}$

Adherence to IPT $^{\$}$

$\begin{array}{lll}\text { Poor } & 1 & 1.74(0.25,12.38) \\ \text { Fair } & 3 & 1.25(0.40,3.87) \\ \text { Good } & 38 & 1.66(1.20,2.27)\end{array}$

Cumulative exposure to ART (years)

$\begin{array}{lll}1-2 & 6 & 13.30(5.99,29.68) \\ 3-5 & 27 & 6.66(4.57,9.71) \\ >5 & 79 & 2.74(2.19,3.41)\end{array}$

Cumulative follow-up time (years)

$\begin{array}{lll}1-2 & 10 & 14.70(7.89,27.25) \\ 3-4 & 25 & 7.05(4.76,10.42) \\ 5-6 & 77 & 2.64(2.11,3.31)\end{array}$

Region

$\begin{array}{lll}\text { Maekel (Central) } & 73 & 3.02(2.39,3.79) \\ \text { Debub } & 11 & 4.88(2.70,8.82) \\ \text { Anseba } & 16 & 5.22(3.19,8.51) \\ \text { Northern Red Sea } & 9 & 9.22(4.79,17.71) \\ \text { Southern Red Sea } & 1 & 1.45(0.20,10.29) \\ \text { Gash-Barka } & 2 & 0.92(0.23,3.69)\end{array}$

Crude rate ratio (95\% CI)

$$
2.31(1.61-3.29)
$$

0.002

Ref

$p$ value

0.002

$\begin{array}{ll}6.89(3.28,11.74) & <0.001 \\ 3.89(2.12,6.71) & <0.001 \\ \text { Ref } & \end{array}$

$1.05(0.03,6.23) \quad 0.618$

$0.75(0.15,2.38) \quad 0.999$

Ref

$\begin{array}{ll}4.87(1.73,11.01) & 0.002 \\ 2.43(1.51,3.81) & <0.001 \\ \text { Ref } & \end{array}$

$5.55(2.56,10.76) \quad<0.001$

$2.67(1.63,4.23) \quad<0.001$

Ref

$\begin{array}{ll}35.82(9.57,30.37) & <0.001 \\ 5.28(1.15,9.09) & 0.022 \\ 5.64(1.32,50.61) & 0.008 \\ 9.97(2.06,94.8) & <0.001 \\ 2.57(0.03,30.13) & 0.564 \\ \text { Ref } & \end{array}$

PLHIV people living with HIV, IPT isoniazid preventive therapy, Ref reference category, $A R T$ antiretroviral therapy ${ }^{*}$ Viral load was categorized as suppressed if the count is $<50$ copies $/ \mathrm{mL}$ and unsuppressed $\geq 50$

${ }^{\$}$ Information for some patients on these covariates was missing

${ }^{\ddagger}$ While computing these variables, we excluded children less than 16 years of age from the analysis $(N=6178)$ as they do not relate to pediatric populations 
Table 6 Risk factors of tuberculosis, in PLHIV attending at all national and regional referral hospitals in Eritrea followed up between November 1, 2014 and November 1, $2020(N=6803)$ using bivariate and multivariate analysis Cox proportional hazard model

\begin{tabular}{|c|c|c|c|c|}
\hline Variables & Unadjusted HR (95\% CI) & $p$ value & Adjusted HR (95\% CI) & $p$ value \\
\hline \multicolumn{5}{|c|}{ Exposure to IPT $\mathrm{IP}^{\#}$} \\
\hline Unexposed & $5.05(3.45,7.39)$ & $<0.001$ & $3.75(2.89,6.13)$ & $<0.001$ \\
\hline Exposed & Ref & & Ref & \\
\hline \multicolumn{5}{|l|}{ Sex } \\
\hline Male & $1.99(1.37,2.88)$ & $<0.001$ & $1.53(0.93,2.51)$ & 0.094 \\
\hline Female & Ref & & Ref & \\
\hline \multicolumn{5}{|c|}{ History of regular alcohol use $\mathrm{P}^{¥}$} \\
\hline Yes & $2.73(1.26,5.94)$ & 0.011 & $2.25(0.91,5.63)$ & 0.081 \\
\hline No & Ref & & Ref & \\
\hline \multicolumn{5}{|c|}{ History of malnutrition } \\
\hline Yes & $8.66(3.79,19.73)$ & $<0.001$ & $5.74(2.14,15.33)$ & $<0.001$ \\
\hline No & Ref & & Ref & \\
\hline \multicolumn{5}{|c|}{ History of imprisonment ${ }^{¥}$} \\
\hline Yes & $2.51(1.21,5.19)$ & 0.013 & $2.22(0.83,5.96)$ & 0.112 \\
\hline No & Ref & & Ref & \\
\hline \multicolumn{5}{|c|}{ CD4 cell count $($ cell $/ \mu \mathrm{L})$} \\
\hline$<200$ & $3.83(2.59,5.65)$ & $<0.001$ & $1.92(1.11,3.32)$ & 0.020 \\
\hline$\geq 200$ & Ref & & Ref & \\
\hline \multicolumn{5}{|c|}{ Viral load suppression $(\text { copies } / \mathrm{mL})^{*}$} \\
\hline Detectable & $1.96(1.28,2.97)$ & 0.002 & $0.66(0.23,1.83)$ & 0.421 \\
\hline Undetectable & Ref & & Ref & \\
\hline \multicolumn{5}{|c|}{ Level of adherence to ART } \\
\hline Poor & $6.33(3.744,10.68)$ & $<0.001$ & $2.17(0.97,4.85)$ & 0.058 \\
\hline Fair & $3.92(2.29,6.72)$ & $<0.001$ & $2.44(1.18,5.02)$ & 0.015 \\
\hline Good & Ref & & Ref & \\
\hline \multicolumn{5}{|c|}{ Cumulative exposure to ART (years) } \\
\hline $1-2$ & $2.97(1.27,6.93)$ & 0.012 & $0.67(0.16,2.91)$ & 0.602 \\
\hline $3-5$ & $2.34(1.49,3.67)$ & $<0.001$ & $1.14(0.63,2.07)$ & 0.665 \\
\hline
\end{tabular}


Table 6 continued

\begin{tabular}{lllll}
\hline Variables & Unadjusted HR (95\% CI) & $p$ value & Adjusted HR (95\% CI) & $p$ value \\
\hline$>5$ & Ref & Ref & \\
\hline
\end{tabular}

PLHIV people living with HIV, IPT isoniazid preventive therapy, Ref reference category

${ }^{*}$ Viral load was categorized as undetectable if the count is $<50$ copies $/ \mathrm{mL}$ and detectable $\geq 50$

${ }^{\#}$ Overall effect of IPT adjusted for all covariates

${ }^{\ddagger}$ While computing these variables, we excluded children less than 16 years of age from the analysis $(N=6178)$ as they are not related to pediatric populations

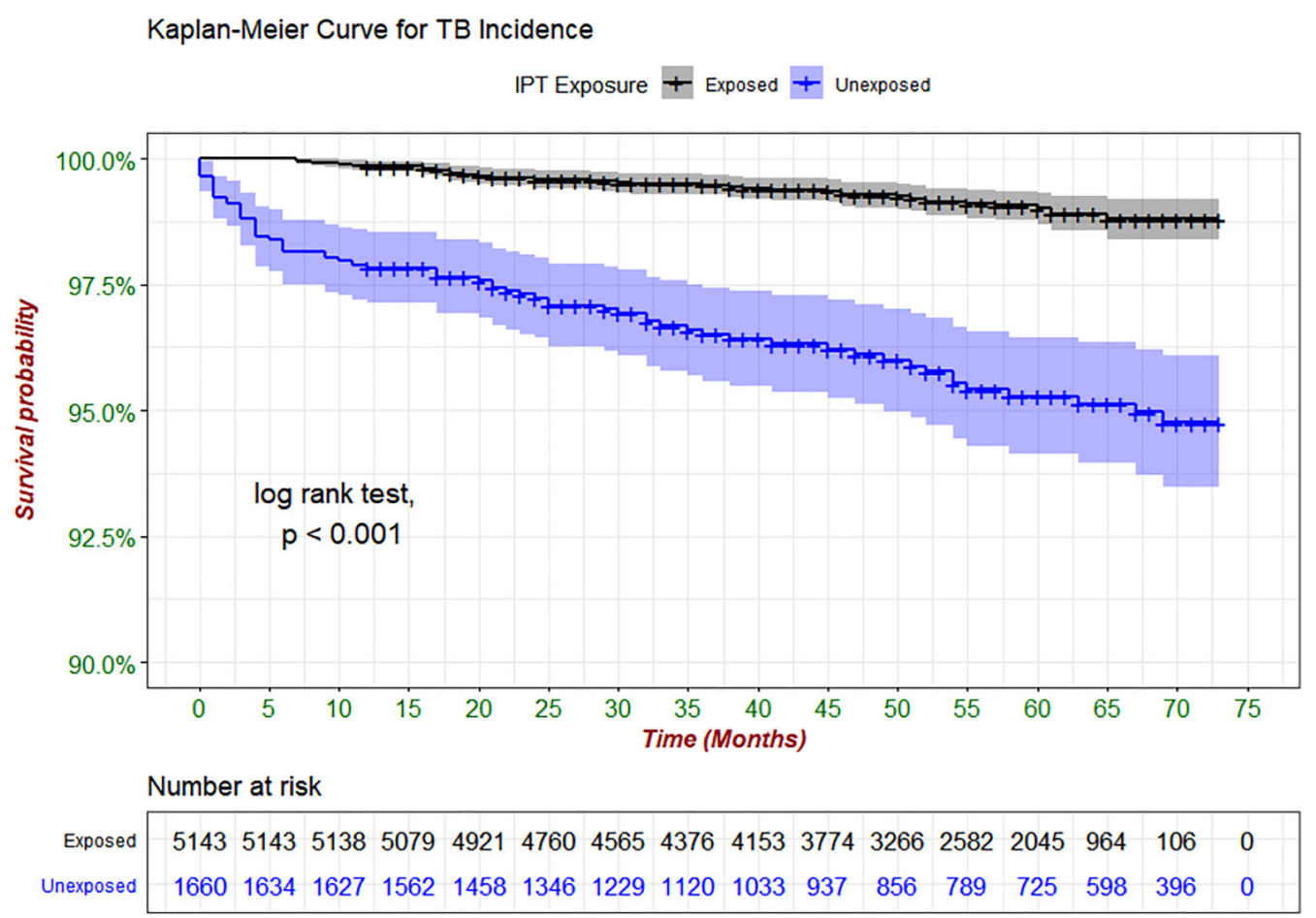

Fig. 4 Kaplan-Meier plot: estimating survival probability versus time without tuberculosis in the isoniazid exposed and unexposed groups using survival regression analysis model

could protect active TB for about 5 years. In the current study, the survival analysis showed that a few patients developed incident TB right after the completion of the 6-month IPT and that the protective effect tended to diminish rapidly after the first year following initiation of IPT. This was consistent with findings reported in countries with a high TB burden [31-36]. In contrast, Golub et al. reported that a 6-month
IPT could reduce the risk of TB for as long as 7 years [28]. The number needed to treat indicated that in order to see a beneficial effect of IPT in one patient, at least 31 PLHIV need to be treated with a 6-month course of IPT which is similar to what was reported elsewhere [30].

Other independent risk factors that affected TB morbidity in the study groups such as CD4 count less than 200 cells $/ \mu \mathrm{L}$, decreased level of 
Table 7 Impact of isoniazid preventive therapy on all-cause and TB-related mortality in PLHIV attending at all national and regional referral hospitals in Eritrea followed up between November 1, 2014 and November 1, $2020(N=6803)$

\begin{tabular}{lllll}
\hline Variables & $\begin{array}{l}\text { Incident cases of } \\
\text { mortality }(\boldsymbol{n})\end{array}$ & $\begin{array}{l}\text { Incidence rate per } \mathbf{1 0 0 0} \text { person-years } \\
(\mathbf{9 5 \%} \mathbf{~ C I})\end{array}$ & $\begin{array}{l}\text { Crude hazard ratio } \\
(\mathbf{9 5 \%} \mathbf{C I})\end{array}$ & $\boldsymbol{p}$ value \\
\hline All-cause mortality & & $2.41(1.85,3.14)$ & $<0.0001$ \\
Unexposed 94 & $18.83(15.41,23.01)$ & Ref & \\
Exposed 134 & $7.94(6.70,9.39)$ & & 0.0157 \\
TB-related mortality & & $4.27(1.32,13.85)$ & \\
Unexposed 7 & 1.45 & Ref & \\
Exposed & 5 & 0.30 &
\end{tabular}

$T B$ tuberculosis, PLHIV people living with HIV, $C I$ confidence interval, $n$ number

adherence to ART, and history of malnutrition were more or less similar to findings of other studies reported elsewhere $[9,37]$. The first four risk factors, directly or indirectly, could make the person susceptible to TB by compromising the immune system, which indicates the importance of lifestyle changes that boost immunity.

Taking all the findings and the inference discussed thereof into account, this study could bring the following programmatic and policy implications: (1) Implementation of a 6-month IPT in a country with a moderate TB burden appears to be effective. As this study only evaluated the beneficial effect of IPT, the risks of the intervention or effectiveness-risk balance and clinical significance of the observed statistically significant survival probability without $\mathrm{TB}$, in the exposed group, need to be systematically analyzed to support the decision context. (2) The protective effect of the intervention tended to diminish after 12 months of the initiation of IPT and warrants attention from policy makers and/or program directors on what to do to ensure patients' long-term protection from TB.

The study had the following limitations: This observational study evaluated effectiveness of a 6-month IPT among PLHIV having at least 1 year of recorded follow-up on either ART only or ART and IPT. Thus, findings of this study could not be generalizable to those who were lost to follow-up or died without having at least 1 year of recorded follow-up. Also, the results could not be generalized to PLHIV who interrupted/could not complete a 6-month isoniazid and those who developed/had TB just before starting ART or IPT. Moreover, there might be situations where the treating physicians prescribed isoniazid preventive therapy mainly to those whom they felt would benefit most such as those with weakened immune system, malnourished, and had close contact with patients with TB. Such potential differential prescribing/ channeling bias, though efforts were made to adjust their effects, might have affected our results. The fact that the majority of the data for the variables used in this study were taken from clinical cards, in itself, could have some implications. For instance, in situations where patients had variable levels of adherence during the study period, the most frequent of those recorded statuses of adherence was taken to represent each subject's adherence. Thus, readers should note that the level of ART adherence reported for some patients might not be the same throughout the study period. Another limitation is that some TB cases were clinically diagnosed; thus, diagnosis could not be validated and that could lead to overestimation of TB cases. Third, it is very difficult to diagnose TB in PLHIV having very low CD4 cell counts $(\leq 100 / \mathrm{mL})$ which requires a lipoarabinomannan test [38]. This diagnostic equipment was not available in Eritrea during the study period and hence some active TB cases in severely ill patients might also have been left undiagnosed. 
As a consequence, the incidence rate of $\mathrm{TB}$ could potentially be underestimated. Lastly, the fact that the cause of death could not be properly qualified in about one-third of the mortalities limited our ability to explore if IPT had an impact on reduction of TB-related mortality. Besides, exclusion of individuals who interrupted their IPT and those having less than 1-year exposure to ART and/or IPT might have also affected the findings on all-cause mortality.

\section{CONCLUSION}

A 6-month course of IPT was found to be effective in reducing incident TB in PLHIV. The protection, nevertheless, appeared to diminish rapidly after 6 months following completion of the intervention. This warrants attention from policy makers and program directors particularly in the need to achieve ways of boosting the 6-month preventive therapy and ensuring prolonged protection of patients. In addition, further studies that assess the risks of the intervention are also required to enable decision makers to evaluate the effectiveness-risk balance of the intervention, which is an important parameter in the decision-making context.

\section{ACKNOWLEDGEMENTS}

The authors would like to sincerely thank the staff of the study sites in general and those involved in data collection in particular. The authors would also like to acknowledge the staff of the Eritrean Medicines and Food Administration: Merhawi Bahta, Merhawi Debesai, Sirak Tefamariam and Yodit Fitsum for their supervisory role during data collection and entry. The authors appreciate pharmacy interns: Abel Tekle, Filipos Yohannes, Heaven Yohannes, Hermon Berhane, Lilay Berhane, Natnael Bereket, Natnael Russom, Saleh Idrisnur and pharmacist Liya Abraham who participated in data entry. Finally, the authors sincerely thank Iyassu Bahta, Director of the National Medicines and Food Administration for his commendable support at all levels of the project and the Communicable Disease Control (CDC) division of the Ministry of Health of the State of Eritrea for their immense cooperation, comments and partial funding of the study.

Funding. This work was funded, in part, by the CDC Division of the Ministry of Health of the State of Eritrea (Global Fund HIV Grantactivity code 9.8). No funding or sponsorship was, however, received for publication of this article. The funding institution had no role in the design, interpretation and write-up of the article.

Authorship. All named authors meet the International Committee of Medical Journal Editors (ICMJE) criteria for authorship for this article, take responsibility for the integrity of the work as a whole, and have given their approval for this version to be published.

Authors' Contributions. Mulugeta Russom, Katia Verhamme and Bruno H. Stricker conceptualized the study. All authors contributed on the design and interpretation of the results. Henok G. Woldu was responsible for the data analysis. Mulugeta Russom supervised the data collection and analysis process and wrote the first draft of the manuscript. All authors critically reviewed the manuscript and provided their consent for publication. The whole research project was made under the supervision and guidance of Katia Verhamme and Bruno H. Stricker and funding acquisition was made by Mulugeta Russom.

Disclosures. Mulugeta Russom, Henok G. Woldu, Araia Berhane, Daniel Y.B. Jeannetot, Bruno H. Stricker and Katia Verhamme have nothing to disclose.

Compliance with Ethics Guidelines. Ethical clearance to conduct the study was obtained from the Health Research Ethics and Protocol Review Committee of the Ministry of Health of the State of Eritrea (reference number 7-18/ 2020). Patients informed/written consent was waived as the study was retrospective. 
Data Availability. All data generated or analyzed during this study are included in this published article/as supplementary information files.

Open Access. This article is licensed under a Creative Commons Attribution-NonCommercial 4.0 International License, which permits any non-commercial use, sharing, adaptation, distribution and reproduction in any medium or format, as long as you give appropriate credit to the original author(s) and the source, provide a link to the Creative Commons licence, and indicate if changes were made. The images or other third party material in this article are included in the article's Creative Commons licence, unless indicated otherwise in a credit line to the material. If material is not included in the article's Creative Commons licence and your intended use is not permitted by statutory regulation or exceeds the permitted use, you will need to obtain permission directly from the copyright holder. To view a copy of this licence, visit http://creativecommons.org/licenses/by$\mathrm{nc} / 4.0 /$.

\section{REFERENCES}

1. Tuberculosis Regional Eastern European and Central Asian Project (TB-REP). WHO Regional Office for Europe 2017. http://www.euro.who.int/_data/ assets/pdf_file/0003/351291/TB_REP_Mid-term_ update_English.pdf?ua=1. Accessed 12 Dec 2020.

2. Sterling T, Pham PA, Chaisson RE. HIV infectionrelated tuberculosis: clinical manifestations and treatment. Clin Infect Dis. 2010;50(Suppl 3): S223-30. https://doi.org/10.1086/651495.

3. Corbett EL, Marston B, Churchyard GJ, et al. Tuberculosis in sub-Saharan Africa: opportunities, challenges, and change in the era of antiretroviral treatment. Lancet. 2006;367(9514):926-37.

4. Houben RM, Dodd PJ. The global burden of latent tuberculosis infection: a re-estimation using mathematical modelling. PLoS Med. 2016;13(10): e1002152.

5. Selwyn PA, Hartel D, Lewis VA. A prospective study of the risk of tuberculosis among intravenous drug users with human immunodeficiency virus infection. N Eng J Med. 1989;320:545-50.
6. World Health Organization. Guidelines for intensified tuberculosis case-finding and Isoniazid preventive therapy for people living with HIV in resource-constrained settings. WHO Geneva; 2011.

7. Ross JM, Badjie A, Rangaka MX, et al. Isoniazid preventive therapy plus antiretroviral therapy for the prevention of tuberculosis: a systematic review and meta-analysis of individual participant data. Lancet HIV. 2021;8(1):E8-15. https://doi.org/10. 1016/S2352-3018(20)30299-X.

8. Kalk E, Heekes A, Mehta U, et al. Safety and effectiveness of Isoniazid preventive therapy in pregnant women living with human immunodeficiency virus on antiretroviral therapy: an observational study using linked population data. Clin Infect Dis. 2020. https://doi.org/10.1093/cid/ciz1224.

9. Tiruneh G, Getahun A, Adeba E. Assessing the impact of isoniazid preventive therapy (IPT) on tuberculosis incidence and predictors of tuberculosis among adult patients enrolled on ART in Nekemte Town, Western Ethiopia: a retrospective cohort study. Interdiscipl Perspect Infect Dis. 2019. https://doi.org/10.1155/2019/1413427.

10. Geremew D, Endalamaw A, Negash M, et al. The protective effect of isoniazid preventive therapy on tuberculosis incidence among HIV positive patients receiving ART in Ethiopian settings: a meta-analysis. BMC Infect Dis. 2019. https://doi.org/10.1186/ s12879-019-4031-2.

11. Semu M, Fenta TG, Medhin G, et al. Effectiveness of isoniazid preventative therapy in reducing incidence of active tuberculosis among people living with HIV/AIDS in public health facilities of Addis Ababa, Ethiopia: a historical cohort study. BMC Infect Dis. 2017. https://doi.org/10.1186/s12879016-2109-7.

12. Ayele HT, Mourik MS, Debray TP, et al. Isoniazid prophylactic therapy for the prevention of tuberculosis in HIV infected adults: a systematic review and meta-analysis of randomized trials. PLoS ONE. 2015;10(11):e0142290. https://doi.org/10.1371/ journal.pone.0142290.t001.

13. Briggs MA, Emerson C, Modi S, Date A. Use of isoniazid preventive therapy for tuberculosis prophylaxis among people living with HIV/AIDS: a review of the literature. J Acquir Immune Defic Syndr. 2015;68(Suppl 3):S297-305. https://doi.org/10. 1097/QAI.0000000000000497.

14. Assebe LF, Reda HL, Wubeneh AD, et al. The effect of isoniazid preventive therapy on incidence of tuberculosis among HIV-infected clients under preART care, Jimma, Ethiopia: a retrospective cohort study. BMC Public Health 2015;15(1):346. https:// doi.org/10.1186/s12889-015-1719-0. 
15. Madhi SA, Nachman S, Violari A, et al. Primary isoniazid prophylaxis against tuberculosis in HIVexposed children. N Engl J Med. 2011;365:21-31. https://doi.org/10.1056/NEJMoa1011214.

16. Mohammed A, Myer L, Wood R, et al. Randomized controlled trial of isoniazid preventive therapy in South African adults with advanced HIV disease. Int J Tuberc Lung Dis. 2007;11(10):1114-20.

17. Zunza M, Gray DM, Young T, et al. Isoniazid for preventing tuberculosis in HIV-infected children. Cochrane Database Syst Rev. 2017. https://doi.org/ 10.1002/14651858.CD006418.pub3.

18. Badje A, Moh R, Gabillard D, et al. Effect of isoniazid preventive therapy on risk of death in west African HIV-infected adults with high CD4 count: long-term follow up of the Temprano ANRS 12136 trial. Lancet Glob Health. 2017;5:e1080-9. https:// doi.org/10.1016/S2214-109X(17)30372-8.

19. Ayieko J, Abuogi L, Simchowitz B, et al. Efficacy of isoniazid prophylactic therapy in prevention of tuberculosis in children: a meta-analysis. BMC Infect Dis. 2014;20(14):91. https://doi.org/10.1186/ 1471-2334-14-91.

20. Ministry of Health of Eritrea. The Sixth Eritrea National HIV/AIDS/STI Strategic Plan: 2021-2026. The State of Eritrea, Ministry of Health. May 2020.

21. UN Data. Eritrea Country profile. http://data.un. org/CountryProfile.aspx/_Images/CountryProfile. aspx?crName=Eritrea. Accessed 1 Apr 2021.

22. UNAIDS. National HIV estimates file. https://www. unaids.org/en/dataanalysis/datatools/spectrumepp. Accessed 1 Apr 2021.

23. Ministry of Health of Eritrea. Comprehensive HIV/ AIDS Care Manual. Ministry of Health, Department of Public Health; 2017.

24. R Core Team. R: A language and environment for statistical computing. R Foundation for Statistical Computing, Vienna, Austria, 2020. https://www.Rproject.org/. Accessed 18 Jan 2021.

25. Russom M, Debesai M, Zeregabr M, et al. Serious hepatotoxicity following use of isoniazid preventive therapy in HIV patients in Eritrea. Pharmacol Res Perspect. 2018. https://doi.org/10.1002/prp2. 423.

26. Russom M, Berhane A, Debesai M, et al. Challenges of hepatotoxicity associated with isoniazid preventive therapy among people living with HIV in Eritrea. Adv Pharmacoepidemiol Drug Saf. 2019. https://doi.org/10.35248/2167-1052.19.8.228.
27. Kerkhoff AD, Kranzer K, Samandari T, et al. Systematic review of TST responses in people living with HIV in underresourced settings: implications for isoniazid preventive therapy. PLoS ONE. 2012;7(11): e49928. https://doi.org/10.1371/ journal.pone.0049928.

28. Golub JE, Cohn S, Saraceni V, et al. Long-term protection from isoniazid preventive therapy for tuberculosis in HIV-infected patients in a mediumburden tuberculosis setting: the TB/HIV in Rio (THRio) study. Clin Infect Dis. 2015;60(4):639-45.

29. Akolo C, Adetifa I, Shepperd S, et al. Treatment of latent infection in HIV infected persons. Cochrane Database Syst Rev. 2010. https://doi.org/10.1002/ 14651858.CD000171.pub3.

30. Rangaka MX, Wilkinson RJ, Boulle A, et al. Isoniazid plus antiretroviral therapy to prevent tuberculosis: a randomised double-blind, placebocontrolled trial. Isoniazid plus antiretroviral therapy to prevent tuberculosis: a randomised doubleblind placebo-controlled trial. Lancet. 2014;384(9944):682-90. https://doi.org/10.1016/ S0140-6736(14)60162-8.

31. Quigley MA, Mwinga A, Hosp M, et al. Long-term effect of preventive therapy for tuberculosis in a cohort of HIV-infected Zambian adults. AIDS. 2001; 15:215-22.

32. Johnson JL, Okwera A, Hom DL, et al. Duration of efficacy of treatment of latent tuberculosis infection in HIV-infected adults. AIDS. 2001;15:2137-47. https://doi.org/10.1097/00002030-20011109000009.

33. Samandari T, Agizew TB, Nyirenda S, et al. 6-month versus 36-month isoniazid preventive treatment for tuberculosis in adults with HIV infection in Botswana: a randomised, double-blind, placebo-controlled trial. Lancet. 2011;377(9777):1588-98.

34. Martinson NA, Barnes GL, Moulton LH, et al. New regimens to prevent tuberculosis in adults with HIV infection. N Engl J Med. 2011;365(1):11-20.

35. Samandari T, Agizew TB, Nyirenda S, et al. Tuberculosis incidence after 36 months' isoniazid prophylaxis in HIV-infected adults in Botswana: a posttrial observational analysis. AIDS. 2015;29(3): 351-9. 0000000000000535 . https://doi.org/10.1097/QAD.

36. Hermans SM, Grant AD, Chihota V, et al. The timing of tuberculosis after isoniazid preventive therapy among gold miners in South Africa: a prospective cohort study. BMC Med. 2016. https:// doi.org/10.1186/s12916-016-0589-3. 
37. Belew H, Wubie M, Tizazu G, et al. Predictors of tuberculosis infection among adults visiting antiretroviral treatment center at east and west Gojjam, northwest Ethiopia, 2017. BMC Infect Dis. 2020. https://doi.org/10.1186/s12879-020-05290-2.

38. Frick M. Report on Tuberculosis Research Funding Trends, 2005-2015: No Time To Lose. New York. Treatment Action Group. 2016. http://www.
treatmentactiongroup.org/sites/default/files/TB FUNDING_2016_WEB.pdf. Accessed 22 July 2020.

\section{Publisher's Note}

Springer Nature remains neutral with regard to jurisdictional claims in published maps and institutional affiliations. 